\title{
Estudio comparativo del espacio aéreo faríngeo según deformidades dentofaciales en radiografías cefalométricas
}

Comparative study of pharyngeal airway space according to dentofacial deformities in cephalometric radiographs

\author{
Julián Mendoza-Lugo ${ }^{1, a}$, Stefany Caballero-García ${ }^{1, b}$, Hugo Ghersi-Miranda ${ }^{c}$
}

\begin{abstract}
RESUMEN
Objetivo: Comparar la dimensión del espacio aéreo faríngeo superior e inferior en las deformidades esqueléticas clase I, II y III determinadas en radiografías cefalométricas. Material y métodos: Se realizó un estudio de tipo retrospectivo donde se analizó 106 radiografías cefalométricas, tomadas en el centro radiográfico del Centro Universitario de Salud de la Universidad Peruana de Ciencias Aplicadas entre los años 2011 y 2014. A través del programa Nemoceph ${ }^{\circledR}$ se marcaron los puntos y trazados cefalométricos principales para determinar la deformidad esquelética (según Steiner) y la dimensión de las vías aéreas superior e inferior (según McNamara). Resultados: La mayor dimensión en promedio $(17,68 \mathrm{~mm})$, se encontró en el espacio aéreo faríngeo superior (EAFS) con en la deformidad dentofacial clase III (DDF clase III) y la menor dimensión en promedio (13,71 $\mathrm{mm}$ ) en la DDF clase II. En el espacio aéreo faríngeo inferior (EAFI), el mayor promedio $(15,98 \mathrm{~mm})$ se presenta en la DDF clase III y el menor promedio $(13,19 \mathrm{~mm})$ en la DDF clase II. Al comparar la dimensión del espacio faríngeo (EAF) entre las DDF, se encontró que existe diferencias estadísticamente significativas entre el EAFS de las DDF II y III, con un valor de $p=0,001$; y en el inferior entre DDF III con DDF I y DDF III con DDFII con valores de $\mathrm{p}=0,0236$ y p $=0,0042$ respectivamente. Conclusiones: En este estudio se encontró que existe diferencias estadísticamente significativas en el espacio aéreo faríngeo superior e inferior entre las tres clases de deformidades esqueléticas.
\end{abstract}

PALABRAS CLAVE: Orofaringe; deformidades dentofaciales; cefalometría; radiografía dental.

\section{SUMMARY}

Objectives: Compare the dimension of the upper and lower pharyngeal airspace between the skeletal deformities class I, II and III in cephalometric radiographs.

Material and methods: A retrospective type of study was made where there were analyzed 106 side radiographies, taken in the X-ray center of the University health Center of the Peruvian University of Applied Sciences UPC between the years 2011 and 2014. Through the program Nemoceph ${ }^{\circledR}$ the main cephalometrics points and tracings were marked to be able to obtain the skeletal deformity (Steiner) and the dimension of the upper and lower airspace (McNamara). Results: In the upper pharyngeal airspace it was found that the highest average dimension was $17.68 \mathrm{~mm}$ founded in the dentofacial deformation class III, and the lowest in class II with a value of $13.71 \mathrm{~mm}$. Fort the lower airspace, the highest average was $15.98 \mathrm{~mm}$ and the lowest $13.19 \mathrm{~mm}$, also founded in skeletal deformation Class III and Class II respectively. While comparing the size of the pharyngeal space between classes of deformity, it was found that there is statistically significant difference between the upper airspace of skeletal deformities class II and III with a value of $p=0.001$; and in the lower, between classes III - I and III - II with values of $p=0.0236$ and $p=0.0042$ respectively. Conclusions: In this study it was found that there is a statistically significant difference in the upper and lower pharingeal airspace between the three dentofacial skeletal deformities.

KEYWORDS: Oropharynx; dentofacial deformities; cephalometry; dental radiography.

${ }^{1}$ Carrera de Odontología, Universidad Peruana de Ciencias Aplicadas. Lima, Perú.

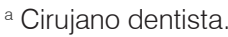

b Docente del área de investigación.

c Cirujano Oral y Maxilofacial, Doctor en Estomatología. 


\section{INTRODUCCIÓN}

Las Deformidades Dento Faciales (DDF), se describen como alteraciones en posición, tamaño y forma de la maxila, mandíbula, dientes y otras estructuras faciales, originados durante la etapa de crecimiento y desarrollo del macizo facial, con influencia de algunos hábitos y funciones nocivas $(1,2,3)$.

Las DDF se clasifican en clase I, II y III, las que pueden ser identificadas por medio de una variedad de cefalogramas, dentro de ellos el de Steiner $(4,5,6)$. La clase I se caracteriza por presentar una buena relación ósea intermaxilar con una alteración solo a nivel de oclusión dental. La clase II esquelética es aquella en la que existe una discrepancia entre la relación maxila-mandíbula con exceso anteroposterior de la maxila, deficiencia en el mismo sentido de la mandíbula o una combinación de ellos y la clase III, frecuentemente asociada a la discrepancia por un exceso anteroposterior de la mandíbula con respecto a maxila o deficiencia en la posición anteroposterior de la maxila con respecto a la mandíbula o ambas $(1,2,7)$.

Para poder realizar un diagnóstico acertado de las DDF, los especialistas en odontología utilizan varias herramientas, dentro de ellas los cefalogramas, los que a partir de puntos craneométricos, se trazan ángulos y distancias con el fin de ubicar en dos planos del espacio la maxila y la mandíbula. Estos estudios pueden aplicarse tanto al tejido óseo como blando, como ejemplo tenemos el análisis de McNamara, que nos permite medir el espacio aéreo faríngeo (EAF) (8).

El EAF se extiende de la base de cráneo al paladar duro (vía de aire), tiene importancia por su relación con la morfología de la cara y los maxilares. Una dimensión reducida del EAF, potencialmente puede repercutir en una respiración nasal difícil, induciendo a una respiración bucal $(1,5)$.

En relación a la dimensión del AEF, existen estudios que demuestran que esta medida varía según el sexo, edad y raza del paciente y está mediado por el desarrollo craneofacial ${ }^{5}$. El conocer y considerar la dimensión del EAF, dentro del análisis dentofacial, sería una ayuda para los odontólogos, en especial a los ortodoncistas y cirujanos maxilofaciales, durante el diagnóstico y planificación de los tratamientos ortoquirúrgicos de las DDF.

El presente estudio tuvo como objetivo el comparar la dimensión del EAF superior e inferior con las DDF clase I, II y III en radiografías cefalométricas mediante los análisis de Steiner y McNamara.

\section{MATERIAL Y MÉTODOS}

Este estudio fue de tipo retrospectivo donde se analizaron 106 radiografías cefalométricas tomadas en el centro radiográfico de la Clínica Docente de la Universidad Peruana de Ciencias Aplicadas (UPC) durante los años 2011 y 2014; la muestra fue determinada utilizando un nivel de confianza del 95\% y una potencia estadística del $80 \%$. Se consideró como criterio de inclusión el sexo y la edad de los pacientes la cual fue entre 9 y 30 años, que no hayan presentado tratamiento quirúrgico u ortodóntico previo. Además, todas las radiografías (RX) fueron tomadas por el equipo ortopantomógrafo de la marca Gendex ${ }^{\circledR}$ de modelo Orthoralix 9200, con un kilovoltaje de $76 \mathrm{Kv}$ y de $7 \mathrm{~mA}$ con un tiempo de exposición de 8 segundos con un factor de aumento de 1,25. Dichas RX, fueron tomadas por el técnico en radiografía oral y maxilofacial, encargado del centro radiográfico, cumpliendo con los siguientes requisitos: plano de Frankfort paralelo al piso, en máxima intercuspidación y con los labios relajados. Se excluyeron las RX que presentaron distorsión y aquellas que no fueron tomadas por el técnico responsable del centro radiográfico, ni en el tiempo determinado. Este estudio se realizó con el consentimiento y aprobación previa del comité de ética de la UPC, registrado con CEI IPI250-15.

Una vez obtenida la base de datos y las RX, se procedió a través del programa Nemoceph ${ }^{\circledR}$ a marcar los puntos y trazados cefalométricos principales, tanto en tejido blando como duro, para poder obtener la DDF (según Steiner) y la dimensión del EAF superior e inferior, según McNamara (8) (figura 1).

Para establecer el tipo de DDF en sentido antero-posterior se realizó el análisis correspondiente según los valores y normas de Steiner. El ángulo ANB en la Clase I: $2^{\circ}$ con una variación de \pm 2 ; Clase III ANB: $<0^{\circ}$ y para la Clase II ANB: $>4^{\circ} 8$

Una vez determinada la DDF, se procedió a agruparlos según el tipo: grupo $1=\mathrm{DDFI}$; grupo $2=\mathrm{DDF}$ II y grupo $3=$ DDFIII. Posteriormente se obtuvo la dimensión del EAF superior e inferior en cada grupo según el análisis de McNamara (8), utilizando el programa Nemoceph ${ }^{\circledR}$ (figuras 1 y 2).

Se analizaron y obtuvieron los valores promedio, máximo, mínimo y desviación estándar de las dimensiones de EAF superior e inferior. El estudio estadístico se realizó con el programa Stata ${ }^{\circledR}$ versión 12.0, utilizando los análisis de Anova y Kruskall Wallis para la comparación de la dimensión del EAF superior e inferior entre las DDF y para la comparación 


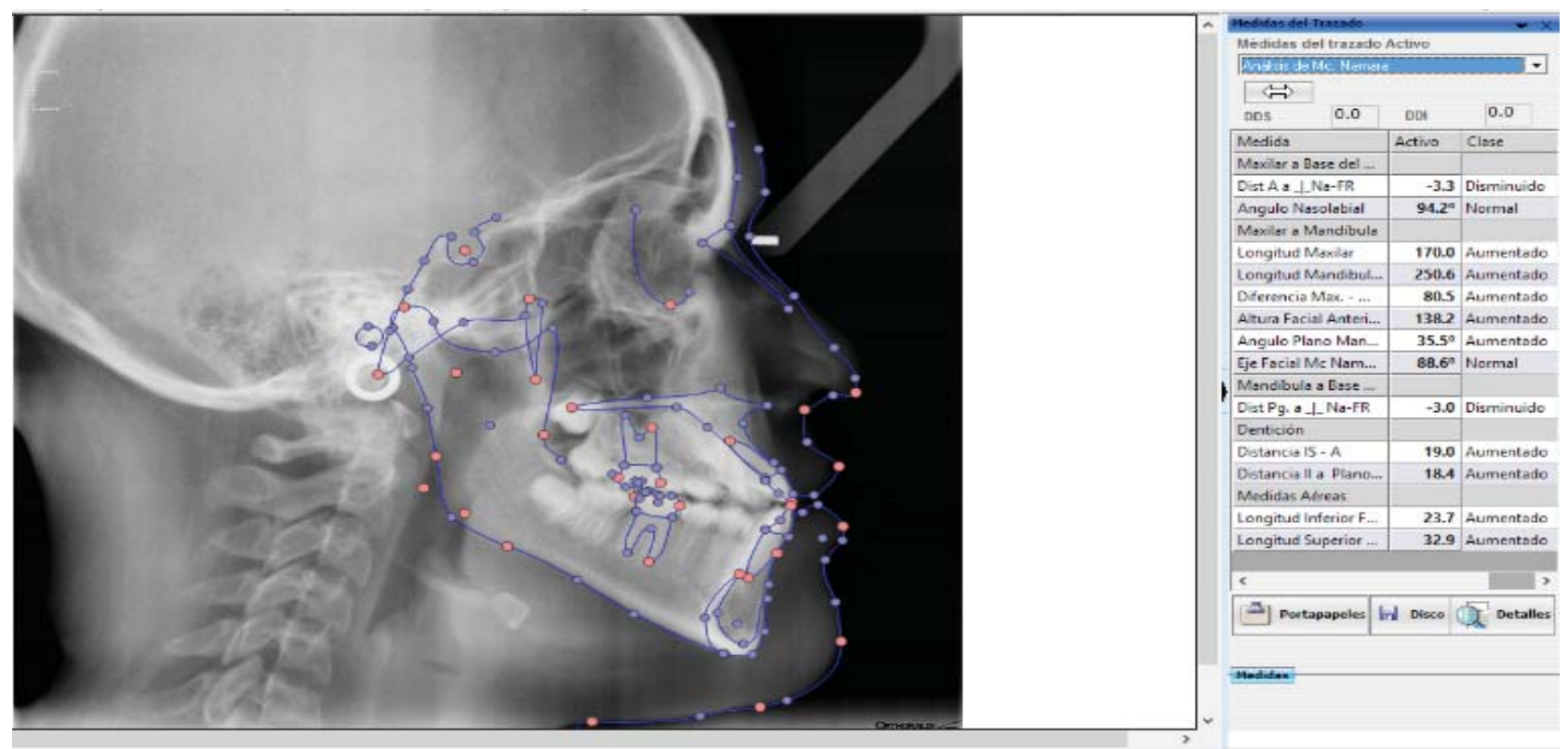

Figura 1. Puntos y trazados cefalométricos principales en tejidos duros y blandos

\begin{tabular}{|c|c|c|}
\hline \multicolumn{3}{|c|}{ Médidas del trazado Activo } \\
\hline \multicolumn{3}{|c|}{ Análisis de Steiner } \\
\hline \multicolumn{3}{|l|}{$\Leftrightarrow$} \\
\hline DDS & DDI & 0,0 \\
\hline Medida & Activo & Unid Desv \\
\hline \multicolumn{3}{|l|}{ Análisis Esqueletal } \\
\hline SNA & $85,8^{\circ}$ & $x$ \\
\hline SNB & $78,1^{\circ}$ & $N$ \\
\hline ANB & $7,7^{\circ}$ & $x x$ \\
\hline SND & $75,7^{\circ}$ & $N$ \\
\hline Distancia SE & 19,3 & $-x$ \\
\hline Distancia SL & 55,9 & $x x$ \\
\hline Angulo del Plano ... & $27,2^{\circ}$ & $x 00$ \\
\hline Angulo del Plano ... & $33,4^{\circ}$ & $\mathrm{N}$ \\
\hline \multicolumn{3}{|l|}{ Análisis Dental } \\
\hline Posición IS & 3,4 & $N$ \\
\hline Posición II & 11,5 & $1000 \times 00$ \\
\hline
\end{tabular}

\begin{tabular}{|c|c|c|c|}
\hline \multicolumn{4}{|l|}{ Medidas del Trazado } \\
\hline \multicolumn{4}{|c|}{ Médidas del trazado Activo } \\
\hline \multicolumn{3}{|l|}{ Análisis de Mc. Namara } & \multirow[t]{2}{*}{$\nabla$} \\
\hline$\Leftrightarrow$ & \multirow[b]{2}{*}{ DDI } & \multirow[b]{2}{*}{0,0} & \\
\hline DDS & & & \\
\hline \multicolumn{2}{|l|}{ Medida } & Activo & Unid \\
\hline \multicolumn{2}{|c|}{ Maxilar a Base del Cráneo } & & \\
\hline \multicolumn{2}{|l|}{ Dist $\mathrm{A}$ a__l_Na-FR } & $-1,2$ & $-x x$ \\
\hline \multicolumn{2}{|l|}{ Angulo Nasolabial } & $113,4^{\circ}$ & $x$ \\
\hline \multicolumn{2}{|l|}{ Maxilar a Mandibula } & & \\
\hline
\end{tabular}

\begin{tabular}{|l|r|l|}
\hline Dentición & & \\
\hline Distancia IS - A & 2,9 & $-x \propto$ \\
\hline Distancia II a Plano A- Pg & 4,7 & $\times \propto$ \\
\hline Medidas Aéreas & & \\
\hline Longitud Inferior Faringe & 7,3 & $-x$ \\
\hline Longitud Superior Faringe & 18,4 & $\mathrm{~N}$ \\
\hline
\end{tabular}

Figura 2. Análisis de Steiner y Mc Namara con el software Nemotec ${ }^{\circledR}$ 
por grupos de DDF se utilizó la prueba post-hoc de Tukey, t de Student y U de Mann Whitney.

\section{RESULTADOS}

Se evaluó 106 radiografías cefalométricas mediante el software Nemotec ${ }^{\circledR} ; 67(63,21 \%)$ del sexo masculino y $39(36,79 \%)$ del sexo femenino con edades entre los 9 y 30 años. Se determinó la distribución de las DDF según el sexo (gráfico 1).

Se determinó el promedio de la dimensión del EAF superior e inferior por sexo, encontrándose que los hombres presentaron un promedio de $14,76 \pm 4,42$ $\mathrm{mm}$ en el superior y $13,62 \pm 3,26 \mathrm{~mm}$ en el inferior. Mientras que para el sexo femenino se encontró una media de $16,53 \pm 4,61 \mathrm{~mm}$ y $14,93 \pm 4,84 \mathrm{~mm}$, con un total de $15,41 \pm 4,55 \mathrm{~mm}$ y $14,1 \pm 3,95 \mathrm{~mm}$ para el superior e inferior respectivamente. Mediante la prueba de t de Student se halló diferencias estadísticamente significativas en el EAF superior $(\mathrm{p}=0.052)$, mas no en el inferior $(p=0.138)$.
Se comparó la dimensión del EAF superior e inferior entre las clases de DDF I, II y III. En el EAF superior se encontró que el mayor promedio de dimensión fue de $17.68 \mathrm{~mm}$ para la DDF clase III y la menor dimensión promedio en la clase II con un valor de 13,71 $\mathrm{mm}$. En cuanto al EAF inferior el mayor promedio fue de $15.98 \mathrm{~mm}$ y el menor de $13,19 \mathrm{~mm}$, también en la DDF clase III y clase II respectivamente. Se encontraron diferencias estadísticamente significativas en ambos casos ( $\mathrm{p}=0,001$ y $\mathrm{p}=0,013$, respectivamente) (tabla 1).

Para determinar las diferencias del EAF superior entre los grupos de DDF se realizó la prueba Post-Hoc de Tukey, encontrándose que existe diferencias estadísticamente significativas entre el EAF de las DDF Clase II y III con un valor de $\mathrm{p}=0,001$. Mientras que al comparar el EAF inferior se realizó las pruebas de $\mathrm{t}$ de Student y U de Mann Whitney, encontrándose diferencias estadísticamente significativas entre las clases de DDF III con I y III con II con valores de $\mathrm{p}=0,0236$ y $\mathrm{p}=0,0042$ respectivamente (tabla 2 ).

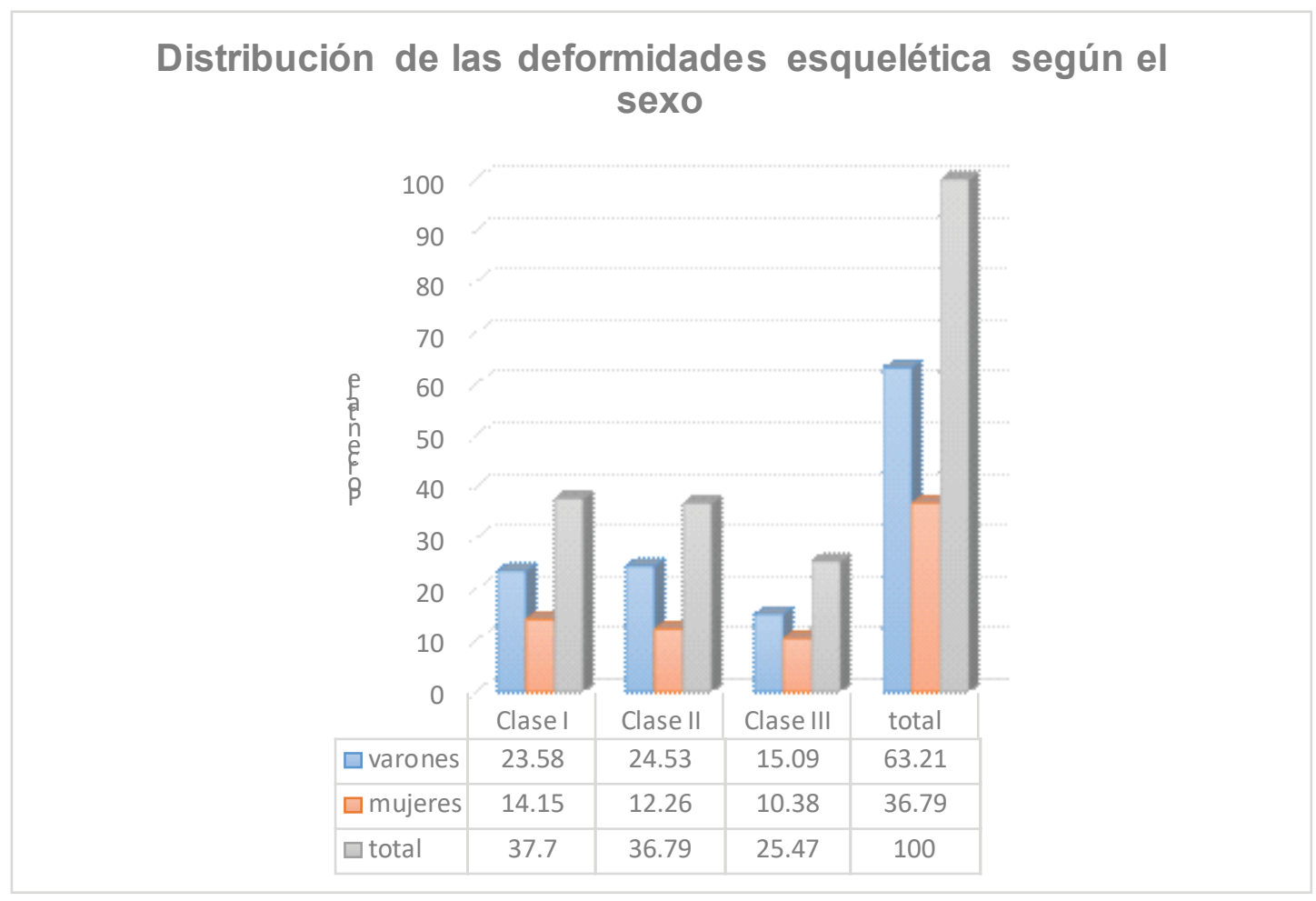

Gráfico 1. Distribución de las deformidades dentofaciales según el sexo. 
Tabla 1. Comparación de la dimensión del espacio aéreo faríngeo superior e inferior entre las clases de deformidad esquelética I, II y III

\begin{tabular}{ccccccc}
\hline $\begin{array}{c}\text { Dimensión del espacio aéreo } \\
\text { faríngeo }\end{array}$ & Deformidad & $\begin{array}{c}\text { Media } \\
(\mathbf{m m})\end{array}$ & D. E & Mínimo & Máximo & P \\
\hline Superior & Clase I & 15,54 & 15,7 & 5,3 & 24,7 & $0,001^{*}$ \\
& Clase II & 13,71 & 12,9 & 7,4 & 19,6 & \\
& Clase III & 17,68 & 17 & 8,4 & 25,1 & \\
\multirow{2}{*}{ Inferior } & Clase I & 13,72 & 13 & 5,5 & 22,2 & $0,013^{* *}$ \\
& Clase II & 13,19 & 12,7 & 5,9 & 21,1 & \\
& Clase III & 15,98 & 14,8 & 10,2 & 30,7 & \\
& & & & & & \\
\hline
\end{tabular}

* Prueba de Anova

**Prueba de Kruskall Wallis

Nivel de significancia estadística, $(\mathrm{p}<0.05)$

Tabla 2. Comparación por grupos la dimensión del espacio aéreo faríngeo superior entre clases de deformidad esquelética clase I, II y III de deformidad esquelética clase I, II y III

\begin{tabular}{cllcc}
\hline $\begin{array}{c}\text { Dimensión del espacio } \\
\text { aéreo faríngeo }\end{array}$ & Grupos & Media & D. E. & P \\
\hline Superior & Clase II - Clase I & $13,71-15,54$ & $12,9-15,7$ & $0,150^{*}$ \\
& Clase III - Clase I & $17,68-15,54$ & $17-15,7$ & $0,119^{*}$ \\
& Clase III - Clase II & $17,68-13,71$ & $17-12,9$ & $0,001^{*}$ \\
Inferior & Clase II - Clase I & $13,19-13,72$ & $12,7-13$ & $0,5213^{* *}$ \\
& Clase III - Clase I & $15,98-13,72$ & $14,8-13$ & $0,0236^{* * *}$ \\
& Clase III - Clase II & $15,98-13,19$ & $14,8-12,7$ & $0,0042^{* * *}$ \\
\hline
\end{tabular}

* Prueba Post-Hoc de Tukey

** Prueba $t$ de Student

*** Prueba U de Mann Whitney

Nivel de significancia estadística $(\mathrm{p}<0.05)$ 


\section{DISCUSIÓN}

El presente estudio comparó las DDF y las dimensiones del EAF, con el objetivo de contribuir en el manejo ortodóntico u ortodóntico-quirúrgico de los pacientes con DDF clase I, II y III.

La comparación de la dimensión del EAF superior e inferior se evaluó mediante el análisis cefalométrico de McNamara en radiografías cefalométricas. Existen estudios similares por distintos autores, sin embargo, consideramos que tener trabajos realizados en nuestra población es importante. En el 2008, Díaz et al., utilizaron el mismo método de interpretación con el objetivo de establecer un índice cefalométrico capaz de predecir la deglución atípica (9). Igualmente, en el 2011, Reddy et al., compararon, los patrones de crecimiento facial con la dimensión del EAF, utilizando el análisis de McNamara (4). Es importante resaltar que los avances en el área de imágenes, actualmente hacen posible obtenerlas en 3D, así como sus dimensiones con mucha exactitud, lo cual aporta mucho a los estudios como lo muestra Sultan et al., (16).

Respecto a los patrones dentofaciales, el presente estudio evaluó y clasificó las DDF en clase I, II y III aplicando el análisis cefalométrico de Steiner, analizando el ángulo $\mathrm{ANB}$, el cual ha sido empleado en diversos estudios $(3,4,10,11)$. En el 2006, Barahona et al., mencionaron los análisis cefalométricos más importantes para el diagnóstico de las DDF, siendo uno de ellos, el de Steiner, utilizando el ángulo ANB como herramienta para ello (3).

Los resultados encontrados para el EAF superior, fueron similares a los obtenidos por Fernández et al., y Arias et al., en donde no hay diferencias estadísticamente significativas en cuanto al sexo, sino en base a otras variables como edad, etnia, biotipo facial y otras características propias de cada estudio $(12,13)$. Esta similitud en los resultados se basa en que existen muchos factores que influyen en la variabilidad de la dimensión del EAF, por ejemplo, el tipo de deformidad esquelética, la altura, la posición de la cabeza, la posición lingual e incluso la intensidad respiratoria fomentan la dilatación o contracción de los músculos de las vías aérea. $(6,14,15)$, sin embargo Rabelo et al., encuentran diferencias, entre mujeres y hombres con DDF clase III en las dimensiones del EAF (16).

El presente estudio además comparó la dimensión del EAF según el tipo de DDF. Los resultados obtenidos muestran que el promedio de mayor dimensión se encontró en la DDF clase III, tanto en el EAF superior e inferior y la menor dimensión se encontró en la clase II, similar a lo hallado por Sultan et al., no se encontraron diferencias estadísticamente significativas en la clase I, esto podría deberse a que es una alteración a nivel dentario, mas no esquelética (16), por lo que no influiría en las estructuras circundantes. Por otro lado, las DDF de Clase III se asocian a una posición más adelantada de la lengua y por la posición del cuello, lo que contribuiría a generar un mayor espacio a nivel de la estructura faríngea. La clase II presenta patrones esqueléticos completamente distintos y algunos autores asocian la respiración oral con DDF Clase II como consecuencia de una reducción en las vías aéreas superiores $(4,5)$.

Dentro de las limitaciones encontradas fue el tipo de población, en donde se evaluaron pacientes con edades entre 9 a 30 años con un promedio de 12 años, por lo que los resultados no pueden ser generalizados a población de cualquier edad. Además, la muestra adquirida y expuesta en la investigación no se excluyó o incluyó radiografías de pacientes que podrían presentar diversos factores asociados que podrían influir en la dimensión del EAF tales como la altura, genética, etnia, etc. Por ello se sugiere realizar más estudios estandarizando la muestra, basándonos en la variabilidad en los resultados encontrados en las investigaciones con respecto a las dimensiones de las vías aéreas superiores en el plano sagital (18), además de utilizar la Tomografía de Haz Cónico por ser más exacta y precisa que las radiografías (19).

Esta investigación ha intentado demostrar que existen diferencias entre la dimensión del EAF superior e inferior con respecto a las DDF Clase I, II y III, con el fin de contribuir en proporcionar parámetros que contribuyan al diagnóstico y manejo de las DDF, especialmente por parte de las especialidades de ortodoncia y cirugía maxilofacial.

\section{CONCLUSIONES}

El promedio de mayor dimensión se encontró en la DDF clase III, tanto en el EAF superior e inferior y la menor dimensión se encontró en la clase II.

Existe diferencias estadísticamente significativas entre el EAF superior de las DDF Clase II y III

No hay diferencias estadísticamente significativas en cuanto al sexo en la dimensión del EAF

Agradecimientos: Se agradece al Instituto de Diagnóstico Maxilofacial (IDM) por la prestación del software Nemotec ${ }^{\circledR}$ para el análisis de las radiografías. 
Conflictos de intereses: Todos los autores declaran no contar con ningún conflicto de intereses en esta investigación.

\section{Correspondencia:}

Stefany Caballero-García

Correo electrónico: pcodccab@upc.edu.pe

\section{REFERENCIAS BIBLIOGRAFICAS}

1. Valle E, Saturno P, Soria V. Evaluación del cumplimiento de los criterios diagnósticos de las deformidades dentofaciales Clase II y III Esqueléticas. Murcia: Universidad de Murcia; 2012.

2. Proffit W, White R. Surgical orthodontic treatment. Boston, Massachusetts: Mosby-Year Book; 1990.

3. Barahona J, Benavides J. Principales análisis cefalométricos utilizados para el diagnóstico ortodonticos. Rev Soc Odontol La Plata. 2006; 1(1): 11-27.

4. Reddy R, Cunduri R, Thomas M, Ganapathy K, Shrikant S. Upper and lower pharyngeal airways in subjects with skeletal Class-I, Class-II \& ClassIII malocclusions and different growth patterns-a cephalometric study. IJCD. 2011; 2(5): 12-8.

5. Dunn GF, Green LJ, Cunat JJ. Relationships between variation of mandibular morphology and variation of nasopharyngeal airway size in monozygotic twins. Angle Orthod. 1973; 43(2): 129-35.

6. Strollo PJ, Rogers RM. Obstructive sleep apnea. N Eng J Med. 1996; 334(2):99-104.

7. Delgado B, Villalpando M. Incidencia de deformidades dentofaciales en un hospital de especialidades. Rev Med IMSS. 2005; 43 (2): 155-9.

8. Porras B, Moya C, Vainer D. Diagnostico ortodóntico: Análisis Cefalométrico. iDental. 2009; 1: 5-26.

9. Días M, Vasconcellos O. Avaliação cefalométrica do espaço orofaríngeo em Pacientes com deglutição atípica. Rev odontociênc. 2008; 23(1):26-30.

10. Canseco J, Gonzales E, De la Torre C. Alteraciones intranasales y nasofaríngeas en pacientes con constricción maxilar y crecimiento vertical de la cara. Rev Cent Am Odontol. 2009; 13(4): 196-204.

11. Marin J, Arango I. Cefalometría comparación y análisis de dos métodos: Steiner y Sassouni. Santafé de Bogotá: Universidad de la Sabana; 2000.

12. Fernandez-Lopez R, Fernandez A, Del Vecchyo C. Estudio comparativo de la permeabilidad de las vías aéreas en pacientes sometidos a cirugía ortognática mandibular. Cirugia Plastica. 2015; 15(1): 5-8.

13. Arias O, Soldevilla L. Valores promedio de la nasofaringe y orofaringe en pacientes con relación esquelética clase I atendidos en la clínica de pregrado de la Facultad de Odontología de la Universidad
Nacional Mayor de San Marcos. Tesis Doctoral. Lima: Universidad Nacional Mayor de San Marcos; 2015.

14. Holmberg H, Linder-Aronson S. Cephalometric radiographs as a means of evaluating the capacity of the nasal and nasopharyngeal airway. Am J Orthod. 2004; 1(3): 479-90.

15. Walsh J, Leigh M, Paduch A. Evaluation of pharyngeal shape and size using anatomical optical coherence tomography in individuals with and without obstructive sleep apnea. J. Sleep Res. 2008; 17: 230-238.

16. Sultan M, Abdulkareem A, Helboub E, et al. Pharyngeal airway spaces in different skeletal malocclusions: a CBCT 3D assessment. Cranio. 2019; 1:1-10. doi: 10.1080/08869634.2019.1583301

17. Rabelo MF, Batista J, Rino-Neto J. Sex differences in pharyngeal airway morphology in adults with skeletal Class III malocclusion. Cranio. 2018; 36(2):98-105.

18. Indriksone I, Jakobsone G. The upper airway dimensions in different sagittal craniofacial patterns: a systematic review. Stomatologija. 2014;16(3):109-17.

19. Nath $M$, Ahmed J, Ongole R, Denny C, Shenoy N. CBCT analysis of pharyngeal airway volume and comparison of airway volume among patients with skeletal Class I, Class II, and Class III malocclusion: A retrospective study. Cranio. 2019 12:1-12. doi: 10.1080/08869634.2019.1652993

Recibido: 16-08-2019

Aceptado: 02-12-2019 